\title{
Magneto-optical response of layers of semiconductor quantum dots and nanorings
}

\author{
O. Voskoboynikov \\ Department of Electronic Engineering and Institute of Electronics, National Chiao Tung University, 1001 Ta Hsueh Road, \\ Hsinchu 300, Taiwan \\ C. M. J. Wijers \\ Faculty of Applied Science, University of Twente, P.O. Box 217, 7500 AE Enschede, The Netherlands \\ J. L. Liu \\ Department of Applied Mathematics, National Chiao Tung University, 1001 Ta Hsueh Road, Hsinchu 300, Taiwan \\ C. P. Lee \\ Department of Electronic Engineering and Institute of Electronics, National Chiao Tung University, 1001 Ta Hsueh Road, \\ Hsinchu 300, Taiwan
}

(Received 17 February 2005; published 30 June 2005)

\begin{abstract}
In this paper a comparative theoretical study was made of the magneto-optical response of square lattices of nanoobjects (dots and rings). Expressions for both the polarizability of the individual objects as their mutual electromagnetic interactions (for a lattice in vacuum) was derived. The quantum-mechanical part of the derivation is based upon the commonly used envelope function approximation. The description is suited to investigate the optical response of these layers in a narrow region near the interband transitions onset, particularly when the contribution of individual level pairs can be separately observed. A remarkable distinction between clearly quantum-mechanical and classical electromagnetic behavior was found in the shape and volume dependence of the polarizability of the dots and rings. This optical response of a single plane of quantum dots and nanorings was explored as a function of frequency, magnetic field, and angle of incidence. Although the reflectance of these layer systems is not very strong, the ellipsometric angles are large. For these isolated dot-ring systems they are of the order of magnitude of degrees. For the ring systems a full oscillation of the optical Bohm-Ahronov effect could be isolated. Layers of dots do not display any remarkable magnetic field dependence. Both type of systems, dots and rings, exhibit an outspoken angular-dependent dichroism of quantum-mechanical origin.
\end{abstract}

DOI: 10.1103/PhysRevB.71.245332

PACS number(s): 73.21.- b, 75.20.Ck, 75.75.+a

\section{INTRODUCTION}

It has been known for a long time that microstructured materials can manipulate electromagnetic radiation. Most of the research in that field focuses at present on photonic crystals, a concept introduced a long time ago. ${ }^{1}$ It is known already that microstructured materials can act as photonic crystals. Recent advances in lithography, colloidal chemistry, and epitaxial growth have made it possible to manufacture artificial meta-materials from semiconductor nano-objects. Further application of these materials in technology demands the extension of the usable frequency range. These demands will push research efforts in this field to the limit. Scale reduction is the classical answer to meet these increased frequency demands and that holds particularly for the new nanostructured metamaterials. When these metamaterials can be made to manipulate electromagnetic fields in the optical range, this will be particularly beneficial for potential applications and devices, as well as for new basic science. The short list of possible implementations being at close range, consists of realization of optical quantum computing, metamaterials with negative refractive index $x^{2,3}$ in the optical region, artificial magnetism in basically nonmagnetic materials ${ }^{4}$ and further.
Semiconductor quantum dots and nanorings are nanosized objects resembling artificial atoms. ${ }^{5,6}$ From these nanoobjects, the nanorings are the newest and they are topologically different from quantum dots since their geometry is nonsimply connected. This different and unique topology gives them unusual magnetic and magnetooptical properties. ${ }^{6}$ The key characteristic of this topology, the center hole, enables trapping of magnetic flux quanta. This property of the nanorings leads to quantum oscillating behavior of the magnetic response of the nanoring for varying magnetic field $\mathbf{B}$, the Aharonov-Bohm (AB) effect. ${ }^{7}$ For a simultaneously applied optical beam this gives rise to the optical $A B$ effects, ${ }^{8,9}$ which can occur only in nanorings. Modification of material properties by means of a magnetic field is an inherent aspect of AB effects, including optical. This option is a prerequisite to make artificial materials, not resembling anything in nature, such as negative refractive index metamaterials. ${ }^{2}$

Up to now, most of the investigations done in the field of magneto-optical effects in nanorings has been about farinfrared (FIR) spectroscopy or magnetophotoluminescence (MP). ${ }^{4,10-13}$ In these methods an additional stimulus has been used, apart from the electromagnetic beam, to determine the response of the rings. These stimuli can be the creation of an extrinsic carrier population (FIR) or an electromagnetic beam of higher frequency (MP). In this sense those methods 


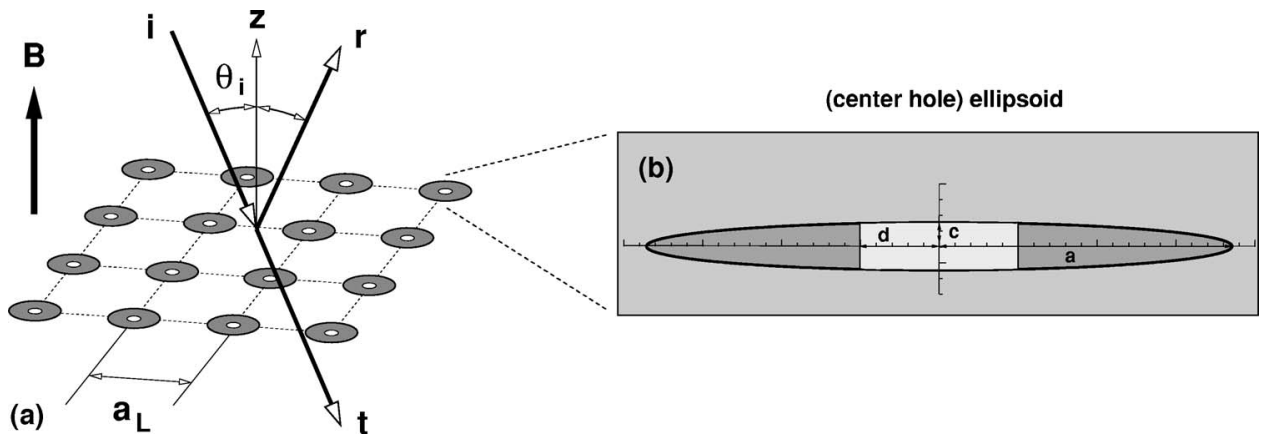

FIG. 1. Schematic diagram of magneto-optical phenomena in a layer of nanorings. $a_{L}$ lattice constant square lattice. Modeling of dots/rings by means of ellipsoids and center hole ellipsoids. $a, c$ long and short axis, $d$ radius cylindrical center hole. $i, r, t$ incoming, reflected, and transmitted beam, respectively. $\theta_{i}$ angle of incidence.

use preexcitation. The data obtained by these methods are very important, but actually only return averaged single nanoring information. For a quantitative characterization of the optical properties of nanoring-based metamaterials a highly developed optical spectroscopy, such as ellipsometry, and an advanced theoretical description are indispensable. Proper understanding and modeling of the collective electromagnetic response of nanoring layers requires a correct approach, taking into account their composite and discrete character. ${ }^{14,16}$ In addition, comparison of the collective magneto-optical response of metamaterials made from semiconductor quantum dots and nanorings can provide important information about the basic physical distinctions between these two types of systems.

\section{THEORY}

In this paper the collective electromagnetic properties of layers of semiconductor quantum dots and nanorings in the optical range will be studied theoretically. It will be shown that the optical $A B$ effect inherent to the ring structures can enrich the optical properties of nanostructured metamaterials. To reach this goal the theory of optical effects will be developed beyond the single (averaged) quantum dot and nanoring picture. It will turn out that, as a result, the magnitude of these effects is clearly within the range of a modern ellipsometric setup.

\section{A. Polarizability: Quantum mechanics}

The systems to be investigated here are two dimensional square lattices of InAs/GaAs quantum dots and nanorings, with lattice parameter $a_{L}$ as shown in Fig. 1. The basic elements of those lattices are dots and "eye" shaped rings as obtained in recent experiments ${ }^{17,18}$ (Fig. 2). For their static electromagnetic response properties these elements will be modeled by means of ellipsoids (for the dots) or center hole ellipsoids (for the rings). This comparative study will use the same outer diameter and aspect ratio for the ellipsoid modeling of both bodies.

Quantum dots and rings are generally classified as artificial atoms. Therefore their optical response should also be described in an atomiclike fashion, e.g., by means of polarizabilities. For atoms such description has been developed originally by Kramers and Heisenberg ${ }^{19}$ and a multitude of derivations and modifications exist of this classical model. Optics in combination with quantum dot and ring structures relies either upon expressions for optical absorption ${ }^{20}$ or for oscillator strengths, ${ }^{21,22}$ being the squared modulus of the optical transition matrix element. ${ }^{9}$ It is not straightforward, however, to transfer the Kramers-Heisenberg expressions to the case of a quantum ring or dot, described by means of envelope functions. To cope with the additional pitfalls we have (re)derived the Kramers-Heisenberg equations for this particular case. ${ }^{23}$ The present commonly used description is only qualitative and the vector character of the electromagnetic response is not well described. Therefore we start this paper with a rehearsal of the main findings in Ref. 23.

To an arbitrary volume element $V$, containing material nanoobjects (dots/rings), an external electric field $\mathbf{E}_{X}(\mathbf{r}, t)$ of frequency $\omega$ is applied. Electromagnetism requires this electric field to be real valued and it has to be described by

$$
\mathbf{E}_{X}(\mathbf{r}, t)=\mathbf{E}_{X}(\mathbf{r}) \cos \omega t=\frac{1}{2}\left[e^{i \omega t}+e^{-i \omega t}\right] \mathbf{E}_{X}(\mathbf{r})
$$

and it has been shown in Ref. 23 that for such field the corresponding dipole strength $\mathbf{d}$ induced in the volume $V$, can be described by

$$
\langle\mathbf{d}\rangle_{V}(t)=\operatorname{Re}\left[\overleftrightarrow{\alpha}_{G}(\omega) \mathbf{E}_{X}(\mathbf{r}) e^{-i \omega t}\right],
$$

where $\alpha_{G}$ is the total monochromatic polarizability. The explicit expression for this monochromatic polarizability $\alpha_{G}$ is given by ${ }^{23}$
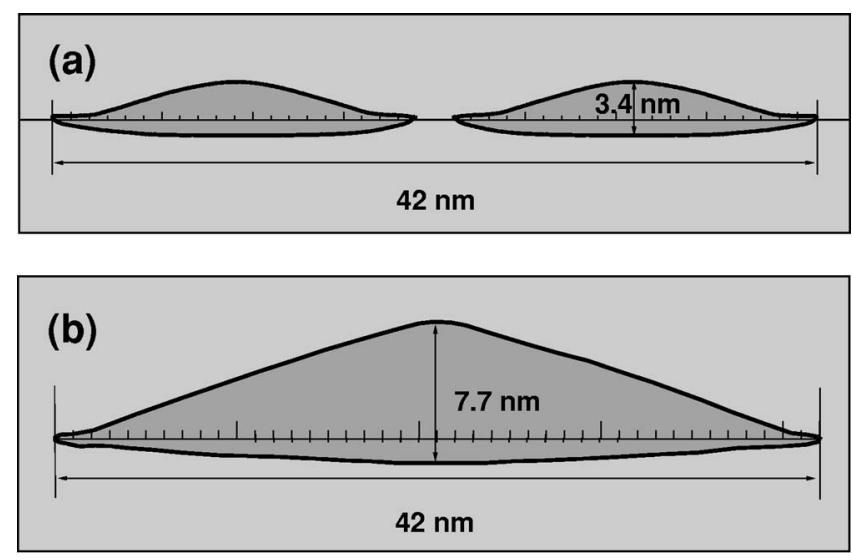

FIG. 2. Typical shape of nanoring and quantum dot structures. (a) Schematic InAs/GaAs "eye" shaped nanoring [after TEM picture (Ref. 17)]. (b) Schematic InAs/GaAs "eye" shaped quantum dot [after TEM picture (Ref. 18)]. 


$$
\begin{gathered}
\overleftrightarrow{\alpha}_{G}(\omega)=\frac{e^{2}}{\hbar} \sum_{l k}\langle k|\mathbf{r}| l\rangle_{V}\langle l|\mathbf{r}| k\rangle_{V}^{T} f_{l k}(\omega) \\
f_{l k}(\omega)=\left(\frac{2 \omega_{l k}}{\omega}\right)\left[\frac{\omega\left[\omega_{l k}^{2}-\omega^{2}-\gamma_{l k}^{2}(\omega)\right]+i \gamma_{l k}(\omega)\left[\omega_{l k}^{2}+\omega^{2}+\gamma_{l k}^{2}(\omega)\right]}{\left[\omega_{l k}^{2}-\omega^{2}-\gamma_{l k}^{2}(\omega)\right]^{2}+4 \omega_{l k}^{2} \gamma_{l k}^{2}(\omega)}\right],
\end{gathered}
$$

where $e$ is the electronic charge and $\langle k|\mathbf{r}| l\rangle$ is the transition matrix element. For each pair of levels $l, k$ we have a transition frequency $\omega_{l k}$ and corresponding damping $\gamma_{l k} \cdot{ }^{23} \mathrm{We}$ have used explicitly that the expression for $\gamma(\omega)$ has to be even in $\omega$. For its specific shape as a function of $\omega$ has been chosen:

$$
\gamma_{l k}(\omega)=\gamma_{l k}\left(\frac{2 \omega_{l k}^{2} \omega^{2}}{\omega_{l k}^{4}+\omega^{4}}\right) .
$$

This expression for $\gamma_{l k}(\omega)$ is just modeling, done such that it is not much different from the traditional $\omega$-independent $\gamma$ in the region of resonance, where $\omega \approx \omega_{l k}$, and resolves the singularity problems in the near static regime, where $\omega \approx 0$. Since it is in general not possible to obtain $\gamma$ in a theoretically hard way, a simple algebraic format has been chosen to model it. Equation (1) can be considered to be the "Swiss army knife" of discrete optics. In a twofold sense it is going to be used in this paper. First we will use it to derive an expression for the polarizability of a nanoring in the enevelope function approximation. Next we will use it to describe the bulk response of the same semiconductor material, the nanoobject is made from. Such relation is necessary to get an independent value for the matrix element controlling the nanoobject polarizability.

We will need for the two different systems, bulk and dot and ring, the following description for the quasiparticle states:

$$
\begin{gathered}
\Psi_{\mathrm{TB}}(\mathbf{r})=e^{i \mathbf{k} \cdot \mathbf{r}} \phi(\mathbf{r}), \text { bulk, } \\
\Psi_{\text {env }}(\mathbf{r})=F(\mathbf{r}) \phi(\mathbf{r}), \text { nanoobject, }
\end{gathered}
$$

where the bulk tight binding function $\phi(\mathbf{r})$ uses the conventional unit cell to define the periodic part of the Bloch states. For InAs the conventional cell consists of four elementary unit cells, each containing one In and one As atom. This cell is cubic and its size is given by the lattice constant $a_{c}$. The bulk wave function has to be normalized over the volume of the conventional unit cell $V_{B}=a_{c}^{3}$, and the envelope function over the volume $V$. This ends up in the conditions

$$
\begin{gathered}
\int_{V_{B}} d \mathbf{r}|\phi(\mathbf{r})|^{2}=1, \\
\int_{V} d \mathbf{r}|F(\mathbf{r})|^{2}|\phi(\mathbf{r})|^{2}=1 .
\end{gathered}
$$

We will need them for any quantitative determination of optical properties.
The first task is to determine the polarizability of a quantum dot or nanoring of volume $V$ in the envelope function approximation. In this approximation the wave functions $\Psi_{u k}(\mathbf{r})$ of the nanoobject have to be written as

$$
\Psi_{u k}(\mathbf{r})=F_{u k}(\mathbf{r}) \phi_{u}(\mathbf{r}),
$$

where $F_{u k}(\mathbf{r})$ is the $k^{\text {th }}$ envelope function of the nanoobject belonging to the $u^{\text {th }}$ Bloch state $\phi_{u}(\mathbf{r})$ of InAs (or any other III-V compound from which the object is made). For $u$ the value $c$ will be used to describe the conduction and $h$ to describe the valence band states, as before. The envelope function $F(\mathbf{r})$ is dimensionless. In what follows the envelope function matrix element $\left\langle F_{u k} \mid F_{u l}\right\rangle_{V}$, will be defined as

$$
\left\langle F_{u k} \mid F_{u l}\right\rangle_{V}=\frac{1}{V_{B}} \int_{V} d \mathbf{r} F_{u k}^{*}(\mathbf{r}) F_{u l}(\mathbf{r})=\delta_{k l} .
$$

The equality follows from combination of both equations in Eq. (3). Now the general expression for the polarizability of an arbitrary volume element (1) needs to be applied to a nanoobject in the envelope function approximation and we need an expression for the optical matrix element $\langle h k|\mathbf{r}| c l\rangle_{V}$. The Bloch states for the electron and hole states will be different and orthogonal. Then we have

$$
\begin{aligned}
\langle h k|\mathbf{r}| \mathrm{cl}\rangle_{V}= & \sum_{i}^{N_{C}} F_{h k}^{*}\left(\mathbf{r}_{i}\right) F_{\mathrm{cl}}\left(\mathbf{r}_{i}\right)\left[\mathbf{r}_{i} \int_{V_{i}} d \mathbf{r}^{\prime} \phi_{h}^{*}\left(\mathbf{r}^{\prime}\right) \phi_{c}\left(\mathbf{r}^{\prime}\right)\right. \\
& \left.+\int_{V_{i}} d \mathbf{r}^{\prime} \phi_{h}^{*}\left(\mathbf{r}^{\prime}\right) \mathbf{r}^{\prime} \phi_{c}\left(\mathbf{r}^{\prime}\right)\right]=\left\langle F_{h k} \mid F_{\mathrm{cl}}\right\rangle_{V} \mathbf{r}_{\mathrm{ch}}, \\
\mathbf{r}_{\mathrm{ch}}= & \int_{V_{B}} d \mathbf{r}^{\prime} \phi_{h}^{*}\left(\mathbf{r}^{\prime}\right) \mathbf{r}^{\prime} \phi_{c}\left(\mathbf{r}^{\prime}\right)=\langle c|\mathbf{r}| h\rangle_{V_{B}},
\end{aligned}
$$

where has been assumed that the entire nanoobject could be split into $N_{C}$ copies $V_{i}$ of the conventional unit cell. Inside these cells $V_{i}$ the envelope function is supposed to be constant. Using the expression for the matrix element (4) we can write the polarizability (1) as

$$
\stackrel{\leftrightarrow}{\alpha}_{G}=\frac{e^{2}}{\hbar} \sum_{h k, \mathrm{cl}}\left|\left\langle F_{h k} \mid F_{\mathrm{cl}}\right\rangle_{V}\right|^{2}\left[\mathbf{r}_{\mathrm{ch}}^{*} \mathbf{r}_{\mathrm{ch}}^{T}\right] f_{h k, \mathrm{cl}}(\omega)
$$

Since the optical matrix elements $\langle c|\mathbf{r}| h\rangle_{V_{B}}$ depend only upon the Bloch states $\phi_{u}$, they do not depend on the indices $k, l$ of the envelope states determined by the geometry of the nanoobjects. Hence, 

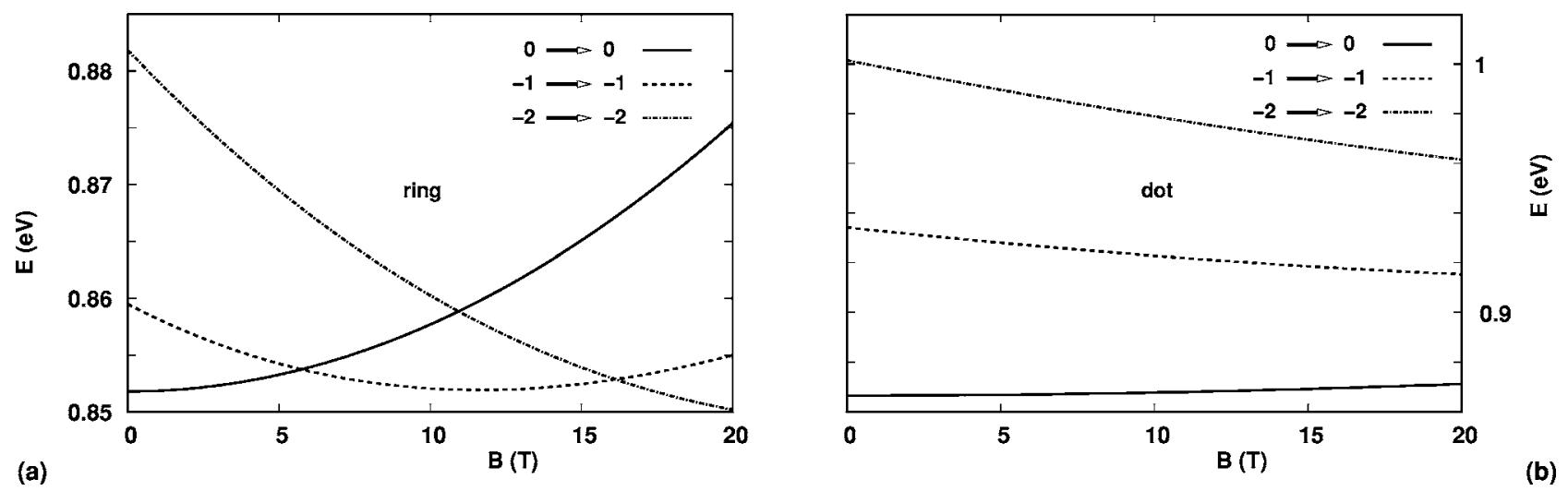

FIG. 3. Transition energy as a function of magnetic field $\mathbf{B}=(0,0, B)$ for $k=l=0,-1,-2$ optical transitions $(k \rightarrow l)$.

$$
\overleftrightarrow{\alpha}_{G}=\frac{e^{2}}{\hbar} \sum_{h, c} \mathbf{r}_{\mathrm{ch}}^{*} \mathbf{r}_{\mathrm{ch}}^{T} \sum_{k, l}\left|\left\langle F_{h k} \mid F_{\mathrm{cl}}\right\rangle_{V}\right|^{2} f_{h k, \mathrm{cl}}(\omega)
$$

To each bulk state $\phi_{u}$ belong $N_{C}$ envelope states. In the calculations to be done further in this paper only a very small number of these envelope states (typically six, three for the heavy hole and three for the electron states) being energetically closest to the energy gap, will be used. This implies that the summation over $c$ involves only the electronic spin $m_{s}$. Such procedure is a good approximation for the imaginary part of the polarizability for energies near the band gap. For energies below this gap the polarizability is predominantly real and involves a summation over the tails of all pairs of states. The result of this summation, however, is almost constant as a function of frequency and can therefore be replaced by a constant value $\alpha_{G S}$. In this approximation we obtain

$$
\overleftrightarrow{\alpha}_{G}(\omega)=\overleftrightarrow{\alpha}_{G S}+\frac{e^{2}}{\hbar} \sum_{h, m_{S}} \mathbf{r}_{\mathrm{ch}}^{*} \mathbf{r}_{\mathrm{ch}}^{T} \sum_{k, l}^{3}\left|\left\langle F_{h k} \mid F_{\mathrm{cl}}\right\rangle_{V}\right|^{2} f_{h k, \mathrm{cl}}(\omega) .
$$

The summation over bulk states is over the indices $h, m_{s}$. For the nanosized objects investigated here, only transitions from heavy hole to electronic Bloch states will be taken into account. The reason is that the dot-ring geometry causes the heavy and light hole states to be energetically different, even in the absence of a magnetic field $\mathbf{B}$.

The polarizability expression (6) embodies an intriguing hybrid of classical and quantum-mechanical thinking. The key ingredients of Eq. (6) are the static term $\alpha_{G S}$ and any dynamic single pair contribution in the summation over $k l$. The $\alpha_{G S}$ dominates the repsonse for frequencies $\omega$ being far below the energy gap $E_{G}$ and is explicitly shape and volume dependent. For any pair $k l$ being at resonance $\left(\hbar \omega \approx E_{k l}\right)$ the polarizability is dominated by this single pair when the nanoobject and damping $\gamma_{k l}$ are small enough (the basic assumption of this paper). Then the polarizability depends only weakly, if at all, on shape and volume (there is a volume and shape effect through the energies $E_{l k}$, but that effect has no influence on the strength of the polarizability as such). Any shape and volume dependence is only in the matrix element $\left\langle F_{h k} \mid F_{c l}\right\rangle_{V}$ and that is in general not much different from 1. This shape and volume insensitivity of the strength of the polarizability under well observable resonance conditions is a fully quantum-mechanical effect and finds its mirror in similar behavior known from conductance quantization ${ }^{24}$. The apparent and remarkable contrast in behavior of the two major components of the polarizability can be exploited to compare directly in one and the same system classical continuous and quantized electromagnetic response.

To determine theoretically the required polarizabilities, asks for calculation of the electron and hole energies and wave functions, adjacent to the energy gap (the edge of optical absorption), for our nanoobjects in the presence of a magnetic field B (see Fig. 3). It was found recently that experimentally relevant simulations of the behavior of the basic elements can only be obtained with three-dimensional models using the experimentally determined shape, strain and composition of the semiconductor nanoobjects. ${ }^{9,11,4}$ In the calculation used here, we assume that the electronic structure of these nanoobjects is governed by the hard-wall confinement potential due to the discontinuity in effective mass parameters over the edges of these objects themselves. This model is commonly used to calculate electron energy states in quantum heterostructures ${ }^{25}$ and allows us to solve the 3D Schrö dinger equation with a minor number of additional approximations. This is particularly useful in this study where we concentrate on the collective optical properties of systems of dots and rings.

Prior to writing the detailed description of the energetic structure of dots and rings we introduce the additional geometric index $i$ :

$$
i=(i),(e)
$$

and where index $u$ will be as defined before. If index $i$ has value $(i)$ it refers to the inner region of the dots and rings and with value $(e)$ to the surrounding embedding matrix. The effective envelope function Hamiltonian is given in the form

$$
\begin{gathered}
\hat{H}=\mathbf{p}_{c} \frac{1}{2 m_{u}(E, \mathbf{r})} \mathbf{p}_{c}+V_{i}(\mathbf{r})+\mu_{B} \frac{g_{u}(E, \mathbf{r})}{2} \sigma \mathbf{B}, \\
\mathbf{p}_{c}=-i \hbar \nabla_{r}+e \mathbf{A}(\mathbf{r}),
\end{gathered}
$$

where $\mathbf{p}_{c}$ stands for the electronic canonical momentum operator, $\nabla_{r}$ is the spatial gradient, and $\mathbf{A}(\mathbf{r})$ is the vector potential $(\mathbf{B}=\boldsymbol{\nabla} \times \mathbf{A})$. For electrons $m_{c}(E, \mathbf{r})$ and $g_{c}(E, \mathbf{r})$ are 
the energy- and position-dependent effective mass and Landé factor, respectively,

$$
\begin{aligned}
\frac{1}{m_{c}(E, \mathbf{r})}= & \frac{2 P^{2}}{3 \hbar^{2}}\left(\frac{2}{E+E_{g}(\mathbf{r})-V_{c}(\mathbf{r})}\right. \\
& \left.+\frac{1}{E+E_{g}(\mathbf{r})-V_{c}(\mathbf{r})+\Delta(\mathbf{r})}\right),
\end{aligned}
$$

and

$$
g_{c}(E, \mathbf{r})=2\left(1-\frac{m_{0}}{m_{c}(E, \mathbf{r})}\left[\frac{\Delta(\mathbf{r})}{3\left[E+E_{g}(\mathbf{r})-V_{c}(\mathbf{r})\right]+2 \Delta(\mathbf{r})}\right]\right),
$$

where $V_{c}(\mathbf{r})$ is the confinement potential, $E_{g}(\mathbf{r})$ and $\Delta(\mathbf{r})$ stand for position-dependent energy band gap and spin-orbit splitting in the valence band, $P$ is the momentum matrix element (Kane parameter), and $\sigma$ is the vector of the Pauli matrices. The free electron mass is $m_{0}$. For the heavy holes $m_{\mathrm{HH}}(E, \mathbf{r})$ and $g_{\mathrm{HH}}(E, \mathbf{r})$ are assumed to be not energy dependent. The hard-wall confinement potential $V_{u}$ is given for both electrons and holes by

$$
\begin{gathered}
V_{u}^{i}(\mathbf{r})=0, \\
V_{u}^{e}(\mathbf{r})=V_{u}^{e} .
\end{gathered}
$$

We consider cylindrically symmetric nano-objects (dots and rings), the shape of which is generated by rotating the contours of Fig. 2 around the $z$ axis. ${ }^{4,9,11,26}$ When the magnetic field is directed along this $z$ axis we can treat the problem in cylindrical coordinates $(\rho, \phi, z)$. The origin of the system is lying in the center of the object.

Because of the cylindrical symmetry of the system the full wave function can be represented as

$$
\begin{gathered}
\Psi(\mathbf{r})=F_{u k}(\mathbf{r}) \phi_{u}(\mathbf{r}), \\
F_{u k}(\mathbf{r})=F_{u k}(\rho, z) e^{i k \phi},
\end{gathered}
$$

where $F_{u k}(\mathbf{r})$ is the envelope function and $\phi_{u}(\mathbf{r})$ is the periodic part of the Bloch state at $\Gamma$ for the semiconductor it belongs to. Labels $k=0, \pm 1, \pm 2, \ldots$, are the orbital quantum numbers for the envelope function, of which only three will be used here for each bulk Bloch state. For the calculations of this paper we need especially detailed expressions for the heavy hole band states $\phi_{l j m_{j}}$ at $\Gamma$ as required by the Kane model. ${ }^{27}$ The quantum number $l$ belongs to the orbital angular momentum, the quantum numbers $j, m_{j}$ determine the total angular momentum. The hole states are

$$
\begin{gathered}
\phi_{1,3 / 2,3 / 2}=\left|h_{3 / 2}\right\rangle=\sqrt{\frac{1}{2}}\left[\left|p_{x}\right\rangle+i\left|p_{y}\right\rangle\right] \uparrow, \\
\phi_{1,3 / 2,1 / 2}=\left|h_{1 / 2}\right\rangle=-\sqrt{\frac{2}{3}}\left|p_{z}\right\rangle \uparrow+\sqrt{\frac{1}{6}}\left[\left|p_{x}\right\rangle+i\left|p_{y}\right\rangle\right] \downarrow, \\
\phi_{1,3 / 2,-1 / 2}=\left|h_{-1 / 2}\right\rangle=-\sqrt{\frac{1}{6}}\left[\left|p_{x}\right\rangle-i\left|p_{y}\right\rangle\right] \uparrow-\sqrt{\frac{2}{3}}\left|p_{z}\right\rangle \downarrow,
\end{gathered}
$$

$$
\phi_{1,3 / 2,-3 / 2}=\left|h_{-3 / 2}\right\rangle=\sqrt{\frac{1}{2}}\left[\left|p_{x}\right\rangle-i\left|p_{y}\right\rangle\right] \downarrow,
$$

where the heavy hole states have $\left|m_{j}\right|=3 / 2$ and the light hole states have $\left|m_{j}\right|=1 / 2 .{ }^{27}$ The electron states in the conduction band are at $\Gamma$ simple product functions

$$
\begin{gathered}
\phi_{0,1 / 2,1 / 2}=\left|c_{\uparrow}\right\rangle=|s\rangle \uparrow, \\
\phi_{0,1 / 2,-1 / 2}=\left|c_{\downarrow}\right\rangle=|s\rangle \downarrow .
\end{gathered}
$$

The quantum number $m_{s}$ determines the projection of the spin along the $z$ axis with values $+\frac{1}{2}(\uparrow),-\frac{1}{2}(\downarrow)$. The chosen geometry determines a 2D Schrödinger equation in the $(\rho, z)$ coordinates

$$
\begin{aligned}
& {\left[-\frac{\hbar^{2}}{2 m_{u}^{i}(E)}\left(\frac{\partial^{2}}{\partial z^{2}}+\frac{\partial^{2}}{\partial \rho^{2}}+\frac{1}{\rho} \frac{\partial}{\partial \rho}-\frac{k^{2}}{\rho^{2}}\right)+\frac{m_{u}^{i}(E) \Omega_{u}^{i 2}(E) \rho^{2}}{8}\right.} \\
& \left.\quad+m_{s} \mu_{B} g_{u}^{i}(E) B+\frac{\hbar \Omega_{u}^{i}(E)}{2} k+V_{u 0}^{i}\right] F_{u k}^{i}(\rho, z) \\
& =E_{u k} F_{u k}^{i}(\rho, z)
\end{aligned}
$$

where we have introduced the cyclotron frequency $\Omega_{u}^{i}(E)$ as

$$
\Omega_{u}^{i}(E)=\frac{e B}{m_{u}^{i}(E)} .
$$

The Ben Daniel-Duke boundary conditions for the problem with the hard-wall potential ${ }^{25}$ can be written as

$$
\begin{gathered}
F_{u k}^{i}[\rho, z(\rho)]=F_{u k}^{e}[\rho, z(\rho)] \\
\frac{1}{m_{u}^{i}(E)}\left[\frac{\partial F_{u k}^{i}[\rho, z(\rho)]}{\partial \rho}\right]=\frac{1}{m_{u}^{e}(E)}\left[\frac{\partial F_{u k}^{e}[\rho, z(\rho)]}{\partial \rho}\right][z=z(\rho)]
\end{gathered}
$$

where $z=z(\rho)$ represents the generating contour for the dots or rings in the $(\rho, z)$ plane. In the expression above we have omitted the explicit reference to electron and hole states for reasons of clarity.

\section{B. Polarizability: Optical matrix element}

In the expression (6) for the total polarizability $\alpha_{G}$ of the nanoobjects in the envelope function approximation a crucial bulk parameter is the optical matrix element $\mathbf{r}_{\mathrm{ch}}$. This matrix element connects the bulk valence and conduction band states at $\Gamma$ as will be worked out here. Despite the advanced state of the field of optoelectronics ${ }^{28-31}$ there is still uncertainty about the correct value of this optical matrix element. For this paper we will use the value given by Eliseev ${ }^{31}$ since it is based upon experimental observations. As will be clear from the following we will have to assign to $r_{\mathrm{ch}}$ the value of $0.60 \mathrm{~nm}$.

The optical response of a III-V semiconductor in the Kane description is governed by transitions between the full states $|h\rangle$ and $|c\rangle$ and not between the basic states $\left|p_{x y z}\right\rangle$ and $|s\rangle$. All transitions starting from the hole states $|h\rangle$ at the top of the (bulk) valence band and ending in the $|s\rangle \uparrow$ state yield three matrix elements 


$$
\begin{gathered}
\mathbf{r}_{3 / 2 \uparrow}=\left\langle e_{\uparrow}|\mathbf{r}| h_{3 / 2}\right\rangle=\sqrt{\frac{1}{2}}[\hat{\mathbf{x}}+i \hat{\mathbf{y}}] r_{v}, \\
\mathbf{r}_{1 / 2 \uparrow}=\left\langle e_{\uparrow}|\mathbf{r}| h_{1 / 2}\right\rangle=-\sqrt{\frac{2}{3}} \hat{\mathbf{z}} r_{v}, \\
\mathbf{r}_{-1 / 2 \uparrow}=\left\langle e_{\uparrow}|\mathbf{r}| h_{-1 / 2}\right\rangle=-\sqrt{\frac{1}{6}}[\hat{\mathbf{x}}-i \hat{\mathbf{y}}] r_{v} .
\end{gathered}
$$

These three elementary matrix elements govern the actual optical response of III-V semiconductors and have to be used in a twofold sense. At first we need them to describe the bulk optical response at the interband transitions onset, where the light and heavy hole band states are degenerate. For the bulk response at the interband transitions onset, Eq. (6) can be used, if we set $F_{h k}=F_{e l}=1$, with $k=l=1$ and let $h$ scan both the heavy and light hole bulk states $\left(m_{j}=\frac{3}{2}, \frac{1}{2},-\frac{1}{2}\right)$. Then all three matrix element contribute equally and Eq. (6) yields effectively a sum of the direct products of the three vectors

$$
\begin{aligned}
\sum_{m_{j}} \mathbf{r}_{m_{j}}^{*} \mathbf{r}_{m_{j} \uparrow}^{T}= & r_{v}^{2}\left[\frac{2}{3} \hat{\mathbf{x}} \cdot \hat{\mathbf{x}}^{T}+i \frac{1}{3} \hat{\mathbf{x}} \cdot \hat{\mathbf{y}}^{T}-i \frac{1}{3} \hat{\mathbf{y}} \cdot \hat{\mathbf{x}}^{T}+\frac{2}{3} \hat{\mathbf{y}} \cdot \hat{\mathbf{y}}^{T}\right. \\
& \left.+\frac{2}{3} \hat{\mathbf{z}} \cdot \hat{\mathbf{z}}^{T}\right] .
\end{aligned}
$$

For transitions to the $|s\rangle \downarrow$ state the $x y$ components change sign. Therefore the total sum of direct vector products over both spin orientations becomes

$$
\begin{gathered}
\sum_{m_{j}, m_{s}} \mathbf{r}_{m_{j}, m_{s}}^{*} \mathbf{r}_{m_{j}, m_{s}}^{T}=r_{\mathrm{ch}}^{2}\left[\hat{\mathbf{x}} \cdot \hat{\mathbf{x}}^{T}+\hat{\mathbf{y}} \cdot \hat{\mathbf{y}}^{T}+\hat{\mathbf{z}} \cdot \hat{\mathbf{z}}^{T}\right] \\
r_{\mathrm{ch}}^{2}=\frac{4}{3} r_{v}^{2} .
\end{gathered}
$$

This is indeed the isotropic kind of response which the bulk of a III-V semiconductor should yield. The quantity $r_{\mathrm{ch}}$ is the bulk real space optical matrix element, as (should be) used elsewhere in the literature.

For the optical response of quantum dots and nanorings the situation is different. The geometry lifts the degeneracy of heavy and light hole states. This means, as mentioned already, that for light hole states the absorption takes place at higher energies than for the heavy hole states. For frequencies being almost at the gap of the nanoring this means that only the first matrix element $\left(m_{j}=\frac{3}{2}\right)$ in Eq. (15) contributes and Eq. (16) now becomes

$$
\sum_{m_{j}} \mathbf{r}_{m_{j} \uparrow}^{*} \mathbf{r}_{m_{j} \uparrow}^{T}=r_{v}^{2}\left[\frac{1}{2} \hat{\mathbf{x}} \cdot \hat{\mathbf{x}}^{T}+i \frac{1}{2} \hat{\mathbf{x}} \cdot \hat{\mathbf{y}}^{T}-i \frac{1}{2} \hat{\mathbf{y}} \cdot \hat{\mathbf{x}}^{T}+\frac{1}{2} \hat{\mathbf{y}} \cdot \hat{\mathbf{y}}^{T}\right]
$$

Incorporating the other spin orientation to this result then yields

$$
\sum_{m_{j}} \mathbf{r}_{m_{j} \uparrow}^{*} \mathbf{r}_{m_{j} \uparrow}^{T}=r_{v}^{2}\left[\hat{\mathbf{x}} \cdot \hat{\mathbf{x}}^{T}+\hat{\mathbf{y}} \cdot \hat{\mathbf{y}}^{T}\right] .
$$

These findings have to be used in Eq. (6) and we have

$$
\overleftrightarrow{\alpha}_{G}(\omega)=\overleftrightarrow{\alpha}_{G S}+\frac{3}{4} \frac{e^{2}}{\hbar} r_{\mathrm{ch}}^{2}\left[\hat{\mathbf{x}} \cdot \hat{\mathbf{x}}^{T}+\hat{\mathbf{y}} \cdot \hat{\mathbf{y}}^{T}\right] \sum_{l=0}^{-2}\left|\left\langle F_{h l} \mid F_{e l}\right\rangle_{V}\right|^{2} f_{h l, e l}(\omega)
$$

The damping term $\gamma_{l k}$ in expression (2) for $\gamma_{l k}(\omega)$ will be chosen to be independent from the indices $l k$ in the near energy gap region. It will be referred to further as $\gamma$ and its value will be chosen such that the experimentally observed linewidth's will be replicated.

\section{Polarizability: Electromagnetism}

The polarizability expressions we have derived until here are derived straight by using Ref. 23 and are purely theoretical. As mentioned in that paper all issues related to electromagnetic self interaction have been discarded. To be of use for experimental work these issues need to be addressed here. This holds also for the relationship between continuum and discrete quantities as used in the hybrid approach of this paper. The discussion should start with the response of a single quantum dot or nanoring. If we use the theoretical polarizability $\alpha_{G}$, exactly as prescribed by Eq. (5), the induced dipole strength $\mathbf{d}$ follows from

$$
\begin{gathered}
\mathbf{d}=\stackrel{\leftrightarrow}{\alpha}_{G} \mathbf{E}_{A}, \\
\mathbf{E}_{A}=\mathbf{E}_{X}+\overleftrightarrow{\mathbf{t}} \cdot \mathbf{d},
\end{gathered}
$$

where $\mathbf{E}_{A}$ is the average electric field over the volume of the dot or ring. It is by definition different from the external field by an amount controlled by the electromagnetic selfinteraction tensor $\mathbf{t}$, as described above. For bodies of revolution with the $z$ axis as axis of revolution this tensor is given in general by

$$
\begin{gathered}
\overleftrightarrow{\mathbf{t}}=\overleftrightarrow{\mathbf{t}}_{S}+\frac{i k^{3}}{6 \pi \epsilon_{0}} \stackrel{\leftrightarrow}{\mathbf{1}}, \\
t_{S, v v}=-\frac{N_{v}(\zeta)}{\epsilon_{0} V}, \\
N_{x}(\zeta)=N_{y}(\zeta)=\frac{1-N_{z}(\zeta)}{2},
\end{gathered}
$$

where $v=x, y, z$. Both the static part $\mathbf{t}_{S}$ and its $k^{3}$ dependent dynamic addition have been obtained already by Lorentz. In the electromagnetic literature, they are commonly known as the (static) Lorentz field and radiative damping term. Since only outgoing waves will be considered to be allowed, a change in sign of $\omega$ will cause also $k$ to change sign. As a result the self interaction tensor turns into its complex conjugate then.

The self interaction tensor is controlled for bodies of revolution by their volume $V$ and depolarization factor $N_{z}$. For ellipsoids with short axis $c$ in the $z$ direction and long axis $a$ in the remaining $x, y$ directions, depolarization factor and volume are given by

$$
N_{z}(\zeta)=\frac{1}{1-\zeta^{2}}\left(1-\frac{\zeta \cos ^{-1} \zeta}{\sqrt{1-\zeta^{2}}}\right)
$$




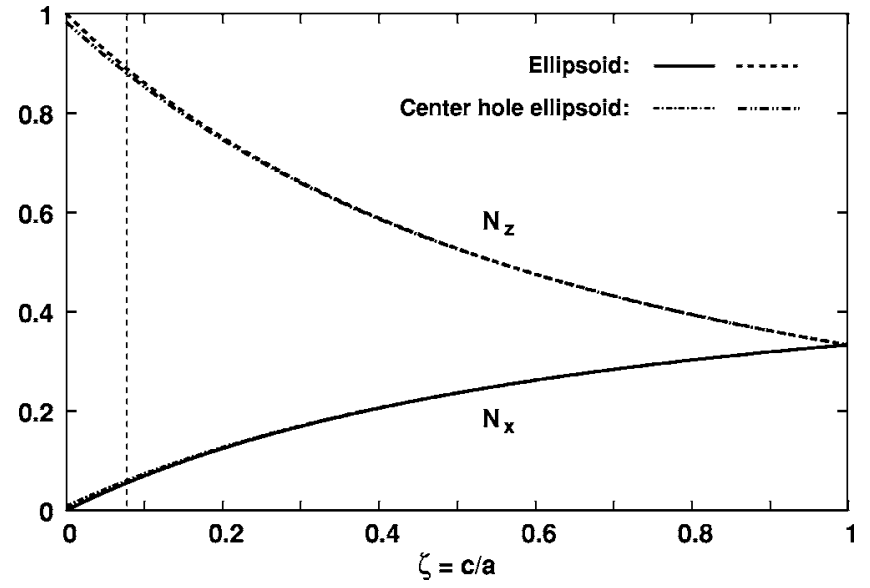

FIG. 4. Depolarization factors $N_{x, z}$ as a function of the aspect ratio $\zeta=c / a$ for normal and center hole ellipsoid. Dashed line at $\zeta$ $=0.081$. Center hole diameter $d=0.257 a$.

$$
V=\frac{4}{3} \pi \zeta a^{3}
$$

where $\zeta=c / a$ is the aspect ratio of the ellipsoid. The Lorentz factor $1 / 3$ is only obtained for the value of $\zeta=1$, corresponding to a sphere. Notice that $N(\zeta)$ is positive. Since $t_{S, z z}$ is negative then, we see that the (average) field inside a dielectric ellipsoid will always be smaller than the external field applied to the ellipsoid, in agreement with the electromagnetism of dielectrics. Along the main axes of the ellipsoid, the electromagnetic self-interaction tensor is diagonal.

The calculation of the electromagnetic self-interaction for the case of a ring-shaped body is a considerable numerical exercise. In the past attempts have been made to derive it for the case of a torus, ${ }^{32}$ but a simple approximate analytical expression can also be obtained by removing a central cylinder with radius $d$ from the ellipsoid treated before. Introducing the angle $\theta_{0}=\sin ^{-1}(d / a)$, we obtain for this case

$$
\begin{aligned}
& N_{z}(\zeta)=\frac{1}{2\left(1-\zeta^{2}\right)}\left\{1+\cos \theta_{0}-\frac{\zeta}{\sqrt{1-\zeta^{2}}}\left[\sin ^{-1}\left(\cos \theta_{0} \sqrt{1-\zeta^{2}}\right)\right.\right. \\
& \left.\left.+\cos ^{-1}(\zeta)\right]\right\} \text {, } \\
& V=\frac{4}{3} \pi \zeta a^{3}\left(1-\frac{d^{2}}{a^{2}}\right)^{3 / 2}
\end{aligned}
$$

The main assumption, apart from the shape chosen, is that the polarization density inside this open ellipsoid has to be constant. Though this is definitely a regula falsi solution, we do not expect much deviation from the correct result for very oblate ellipsoids. The depolarization factors for both types of ellipsoid are shown in Fig. 4. The depolarization factor hardly depends on the presence of the center hole.

For the case that we want to measure the polarizability of a single dot or ring, we have to proceed differently. For the experimental situation we have to use

$$
\mathbf{d}=\stackrel{\leftrightarrow}{\alpha \mathbf{E}_{X}}
$$

where this experimental polarizability $\alpha$ embodies also the electromagnetic self-interaction. Combining properly the two prescriptions for the induction (21) and (25) leads to

$$
\stackrel{\leftrightarrow}{\alpha}^{-1}=\overleftrightarrow{\alpha}_{G}^{-1}-\overleftrightarrow{\mathbf{t}}
$$

This is a very important relationship, showing that there should be a serious discrepancy between measured and quantum mechanically calculated polarizabilities.

In expression (20) for $\alpha_{G}$ we need separately an expression for $\alpha_{G S}$, the quantum-mechanical static polarizability tensor of the dot or ring. For the special case that we represent the dot by an strongly oblate homogeneous dielectric ellipsoid with relative dielectric constant $\epsilon$, this static polarizability is isotropic and can easily be obtained. It suffices to introduce for this ellipsoid its $\alpha_{G S}$ as

$$
\begin{gathered}
\alpha_{G S, v v}=\alpha_{G S}=\epsilon_{0} V(\epsilon-1)=\alpha_{0} f_{V}(\epsilon-1), \\
\alpha_{0}=4 \pi \epsilon_{0} a_{L}^{3}, \\
f_{V}=\frac{\epsilon_{0} V}{\alpha_{0}}=\frac{V}{4 \pi a_{L}^{3}},
\end{gathered}
$$

where we introduce the two normalization factors $\alpha_{0}$ and $f_{V}$. Next we use Eq. (26) to determine the measurable polarizability $\alpha_{S}$ :

$$
\alpha_{S, v v}=\frac{\alpha_{G S}}{1-t_{S, v v} \alpha_{G S}}=\epsilon_{0} V\left[\frac{\epsilon-1}{1+N_{v}(\epsilon-1)}\right] .
$$

The same expression has been obtained along a different line of derivation by Avelin. ${ }^{33}$ For arbitrary shapes of the dots and rings only a full numerical calculation can be done. The result of such calculation will be $\alpha_{S, v v}$. In Eq. (20) we need $\alpha_{G S, v v}$ and not $\alpha_{S, v v}$. The expression for $\alpha_{G S, v v}$ is simple and given already in Eq. (27). The numerical determination of $\alpha_{S, v v}$ is necessary to obtain the right value for $\mathbf{t}_{S}$ for arbitrarily shaped bodies from the relation

$$
\overleftrightarrow{\mathbf{t}}_{S}=\stackrel{\leftrightarrow}{\alpha}_{G S}^{-1}-\stackrel{\leftrightarrow}{\alpha}_{S}^{-1}
$$

This value for $t_{S, v v}$ determines the expression for the applied field in case of arbitrarily shaped bodies.

If we surround this individual dot or ring by a (square) lattice of identical dots and rings, with $a_{L}$ as lattice spacing, the only difference is that we have to replace the external field $\mathbf{E}_{X}$ by the local field $\mathbf{E}_{L}$, given for the single lattice plane by

$$
\begin{aligned}
& \mathbf{E}_{L}=\mathbf{E}_{X}+\overleftrightarrow{\mathbf{f}^{\prime}} \cdot \mathbf{d}, \\
& \mathbf{E}_{A}=\mathbf{E}_{L}+\overleftrightarrow{\mathbf{t}} \cdot \mathbf{d},
\end{aligned}
$$

where $\mathbf{f}^{\prime}$ is the intraplanar transfer tensor for the plane. This transfer tensor $\mathbf{f}^{\prime}$ can be determined numerically to any precision using the Ewald one-fold integral transform. ${ }^{15}$ Vlieger, however, has derived, after much effort, a more concise expression: ${ }^{16}$ 


$$
\begin{aligned}
& {\stackrel{\leftrightarrow}{\mathbf{f}^{\prime}}}^{=} \alpha_{0}^{-1}{\stackrel{\leftrightarrow}{\mathbf{f}^{\prime}}}_{S}+\frac{i}{2 \epsilon_{0} a_{L}^{2}\left|k_{z}\right|}\left[k^{2} \stackrel{\leftrightarrow}{\mathbf{1}}-\mathbf{k}_{\|} \mathbf{k}_{\|}^{T}-k_{z}^{2} \hat{\mathbf{z}} \cdot \hat{\mathbf{z}}^{T}\right]-\frac{i k^{3}}{6 \pi \epsilon_{0}} \stackrel{\leftrightarrow}{\mathbf{1}}, \\
& \mathrm{f}_{S, x x}^{\prime}=\mathrm{f}_{S, y y}^{\prime}=-4.51681, \\
& \mathrm{f}^{\prime}{ }_{S, z z}=-2 \mathrm{f}_{S, x x}^{\prime}=9.03362,
\end{aligned}
$$

where parallel $(\|)$ means parallel to the plane of the dots or rings. The strong aspect of the Vlieger expression is that it gives the intraplanar transfer tensor as a dynamical correction to its static counterpart. This guarantees smooth linkage to the static result, being for a square lattice essentially only the numbers given above. The induction for a dot or ring inside a lattice now becomes

$$
\begin{gathered}
\mathbf{d}=\overleftrightarrow{\alpha}_{G}\left[\mathbf{E}_{X}+\left(\overleftrightarrow{\mathbf{f}^{\prime}}+\overleftrightarrow{\mathbf{t}}\right) \mathbf{d}\right] \\
\left(\overleftrightarrow{\mathbf{f}^{\prime}}+\overleftrightarrow{\mathbf{t}}\right)=\alpha_{0}^{-1}\left({\overleftrightarrow{\mathbf{f}^{\prime}}}_{S}+\overleftrightarrow{\mathbf{t}}_{S}\right)+\frac{i}{2 \epsilon_{0} a_{L}^{2}\left|k_{z}\right|}\left[k^{2} \stackrel{\mathbf{1}}{-} \mathbf{k}_{\|} \mathbf{k}_{\|}^{T}-k_{z}^{2} \hat{\mathbf{z}} \cdot \hat{\mathbf{z}}^{T}\right] \\
\mathrm{t}_{S, v v}=-\frac{N_{v}(\zeta)}{f_{V}}
\end{gathered}
$$

where, as in the Vlieger derivation, the radiation damping term has disappeared.

\section{Electromagnetic response}

The electromagnetic response of the layer of dots/rings, has to be determined in two steps. The dipole strength d induced in the plane is obtained from

$$
\mathbf{d}=\left[\overleftrightarrow{\mathbf{1}}-\overleftrightarrow{\alpha}_{G}\left(\stackrel{\leftrightarrow}{\mathbf{f}^{\prime}}+\overleftrightarrow{\mathbf{t}}\right)\right]^{-1} \overleftrightarrow{\alpha}_{G} \mathbf{E}_{X}
$$

The reflected electric fields at a remote site $\mathbf{R}$ are now given by

$$
\begin{gathered}
\mathbf{E}_{R}(\mathbf{R})=\stackrel{\leftrightarrow}{\mathbf{f}}_{R} \mathbf{d}, \\
\overleftrightarrow{\mathbf{f}}_{R}=\frac{i k^{2} e^{i \underline{\mathbf{k}} \cdot \mathbf{R}}}{2 \epsilon_{0} a_{L}^{2} \beta k_{z}}\left[\stackrel{\leftrightarrow}{\mathbf{1}}-\underline{\hat{\mathbf{k}}} \cdot \underline{\hat{\mathbf{k}}}^{T}\right], \\
\underline{\mathbf{k}}=\mathbf{k}_{\|}-k_{z} \hat{\mathbf{z}},
\end{gathered}
$$

where $\mathbf{f}_{R}$ is the remote interplanar transfer tensor. Physically only those electric fields make sense which go in a direction away from the sources. This means that when the sign of $\omega$ is changed also the corresponding wave number or wave vector has to change sign. Therefore it is easily seen that in the above expression when $\omega$ is replaced by $-\omega$, both $k$ and $k_{z}$ have to change sign. Therefore all transfer tensors $\mathbf{t}, \mathbf{f}^{\prime}$, and $\mathbf{f}_{R}$ will always turn into their complex conjugate when $\omega$ changes sign. We have shown already in detail that the same behavior applies also to $\alpha_{G}{ }^{23}$ This mathematical property guarantees that the dipole strength induced in the plane and the electric field emitted by the plane of dots and rings will be real according to

$$
\mathbf{E}(\mathbf{r}, t)=\operatorname{Re}\left[\overleftrightarrow{\mathbf{f}}_{R}(\omega) \mathbf{d}(\omega) e^{-i \omega t}\right] .
$$

This elementary result and Eqs. (33) and (34) allow the entire electromagnetic derivation to be done using a single complex exponential. Also the following relationship is useful, if not indispensable:

$$
\begin{aligned}
\langle A B\rangle & =\frac{1}{T} \int_{0}^{T} d t A(t) B(t)=\frac{1}{T} \int_{0}^{T} d t \operatorname{Re}\left[\widetilde{A}_{0} e^{-i \omega t}\right] \operatorname{Re}\left[\widetilde{B}_{0} e^{-i \omega t}\right] \\
& =\frac{1}{2} \operatorname{Re}\left[\widetilde{A}_{0}^{*} \widetilde{B}_{0}\right]
\end{aligned}
$$

for any two harmonic fields $A(t), B(t)$ and further $\omega T=2 \pi$. For electromagnetic derivations, different from the quantummechanical derivations treated in Ref. 23 , the complex notation is only auxiliary in character and allows for the common smooth usage.

In a further straightforward manner it is easy to show now that only the following reflection and transmission coefficients suffice to describe the full electromagnetic response of a square plane of dots and rings:

$$
\begin{gathered}
r_{s s}=\frac{f_{k}}{A_{y} \cos \theta_{i}-f_{k}}, \\
r_{p p}=\frac{f_{k} \cos \theta_{i}}{A_{x}-f_{k} \cos \theta_{i}}-\frac{f_{k} \sin ^{2} \theta_{i}}{A_{z} \cos \theta_{i}-f_{k} \sin ^{2} \theta_{i}}, \\
t_{s s}^{-}=1+r_{s s}, \\
t_{p p}^{-}=\frac{f_{k} \cos \theta_{i}}{A_{x}-f_{k} \cos \theta_{i}}-\frac{A_{z} \cos \theta_{i}}{A_{z} \cos \theta_{i}-f_{k} \sin ^{2} \theta_{i}},
\end{gathered}
$$

where $\theta_{i}$ is the angle of incidence. Further use has been made of the notation $t^{-}$for the transmission coefficient to distinguish it from the self-interaction $t$ and of the following abbreviations to make the expressions more concise:

$$
\begin{gathered}
A_{v}=\alpha_{0} \alpha_{G, v v}^{-1}-\left(\mathbf{f}_{S, v v}^{\prime}+\mathbf{t}_{S, v v}\right), \\
f_{k}=2 \pi i a_{L} k
\end{gathered}
$$

with $v$ as defined before. These reflection coefficients are not directly measurable as they are. Measurable are only the reflectances $R_{q q}$ and transmittances $T_{q q}$ defined as

$$
\begin{aligned}
& R_{q q}=r_{q q}^{*} r_{q q}, \\
& T_{q q}=t_{q q}^{-*} t_{q q}^{-},
\end{aligned}
$$

where $q$ stands for the ellipsometric directions $s, p$. From the energy balance point of view these quantities determine the absorbance $A_{q q}$ in the lattice plane according to

$$
A_{q q}=1-R_{q q}-T_{q q}=\frac{\omega \mu_{0} c}{a_{L}^{2} E_{X}^{2} \cos \theta_{i}} \operatorname{lm}\left(\mathbf{E}_{A}^{* T} \mathbf{d}\right),
$$

where $E_{X}$ is the amplitude of the externally incoming plane wave. Measurable, but less directly, are also the ellipsometric angles $\Psi, \Delta$, which follow from the commonly used definition: 
TABLE I. Basic input parameters for lattices of dots and rings. For the meaning of the symbols, see text.

\begin{tabular}{ccc}
\hline \hline & Quantum dot & Nanoring \\
\hline$a_{L}$ & $80.0 \mathrm{~nm}$ & $80.0 \mathrm{~nm}$ \\
$a$ & $18.45 \mathrm{~nm}$ & $18.45 \mathrm{~nm}$ \\
$c$ & $1.49 \mathrm{~nm}$ & $1.49 \mathrm{~nm}$ \\
$d$ & $0.00 \mathrm{~nm}$ & $4.75 \mathrm{~nm}$ \\
$\left|\left\langle F_{h 0} \mid F_{c 0}\right\rangle_{V}\right|$ & 0.9454 & 0.9 \\
$\left|\left\langle F_{h,-1} \mid F_{c,-1}\right\rangle_{V}\right|$ & 0.9285 & 0.9 \\
$\left|\left\langle F_{h,-2} \mid F_{c,-2}\right\rangle_{V}\right|$ & 0.9120 & 0.9 \\
$r_{\mathrm{ch}}$ & $0.60 \mathrm{~nm}$ & $0.60 \mathrm{~nm}$ \\
$\epsilon$ & 12.2 & 12.2 \\
$E_{G}\left(T=0{ }^{\circ} \mathrm{K}\right)$ & $0.42 \mathrm{eV}$ & $0.42 \mathrm{eV}$ \\
\hline \hline
\end{tabular}

$$
\rho_{r}=\frac{r_{p p}}{r_{s s}}=\tan \Psi e^{i \Delta}
$$

In the next section these experimentally accessible quantities will be calculated numerically for the plane of dots and rings.

\section{NUMERICAL RESULTS}

Using the above expressions for reflection coefficients and related ellipsometric angles $\Psi, \Delta$, we have determined the optical response of a square lattice made from nanorings and, for comparison, from quantum dots. All relevant input material has been collected in Table I.

The optical response for individual dots and rings is completely controlled by the polarizability $\alpha_{G}$, given by Eq. (20). The static part of it, $\alpha_{G S}$, is given by Eq. (27) and is completely determined by the volume $V$. Volume $V$ is given for the ellipsoid by Eq. (23) and for the center hole ellipsoid by Eq. (24). The geometrical data determining these ellipsoids (see Fig. 1) are $a, c$, and $d$ and have the values given in Table I. The aspect ratio $\zeta$ for both ellipsoids and center hole ellipsoids then becomes

$$
\zeta=c / a=0.081
$$

and the corresponding depolarization factors $N_{z}, N_{x}$ are for the ellipsoids (dots):

$$
N_{z}=0.8848, \quad N_{x}=0.0576
$$

and for the center hole ellipsoids (rings)

$$
N_{z}=0.8757, \quad N_{x}=0.0622 .
$$

The volume of the ellipsoid (dot) is $V=2.125 \times 10^{-24} \mathrm{~m}^{3}$ and for the center hole ellipsoid (ring) $V=1.917 \times 10^{-24} \mathrm{~m}^{3}$. To calculate the static polarizability $\alpha_{G S}$ we need the value of the dielectric constant $\epsilon$ for (InAs), the material making up the dot or ring and given in Table I. That number belongs to high-frequency (IR) data at room temperature, since we consider that to be the best choice.

For an single isolated dot or ring, using Eq. (28), we find the following tensor components $\alpha_{G S}$ and $\alpha_{v v}$ for dots:

$$
\alpha_{G S}=3.711 \times 10^{-3} \alpha_{0},
$$

$$
\begin{aligned}
& \alpha_{S, x x}=2.256 \times 10^{-3} \alpha_{0}, \\
& \alpha_{S, z z}=3.401 \times 10^{-4} \alpha_{0},
\end{aligned}
$$

and correspondingly for rings

$$
\begin{aligned}
& \alpha_{G S}=3.348 \times 10^{-3} \alpha_{0}, \\
& \alpha_{S, x x}=2.188 \times 10^{-3} \alpha_{0}, \\
& \alpha_{S, z z}=3.434 \times 10^{-4} \alpha_{0},
\end{aligned}
$$

where $\alpha_{0}=5.69677 \times 10^{-32} \mathrm{~F} \mathrm{~m}^{2}$. The anisotropy of the static polarizability $\alpha_{S}$ is entirely due to the electromagnetic selfinteraction of the dot or ring. Quantum mechanics plays no role in it.

This will change for frequencies near the energy gap of the dot or ring. Then there is a strong quantum-mechanical contribution to the anisotropy. To determine numerically the dynamic quantum-mechanical part of the polarizability $\alpha_{G}$ requires knowledge of the overlap matrix elements $\left\langle F_{h l} \mid F_{\mathrm{cl}}\right\rangle$. Those were obtained numerically by solving the effective mass Hamiltonian (13) with the Ben Daniel-Duke boundary condition (14). The resulting values are in Table I . There is also the bulk optical matrix element $r_{\text {ch }}$. The transition frequencies $\omega_{l k}$ are shown in Fig. 3, but the damping $\gamma$ can only be used as a free parameter. We have used two values $2 \mathrm{meV}$ and $5 \mathrm{meV}$ for it. If we take $\hbar \omega=0.86 \mathrm{eV}$ as a typical energy for the interband transitions of the dots or rings, this corresponds to a wavelength $\lambda=1459 \mathrm{~nm}$, well beyond the lattice spacing $a_{L}$. This ensures that the Vlieger expressions can be used as they are and that we do not have to bother about possible higher order reflections.

The ellipsometric angles $\Psi$ and $\Delta$ are shown for this configuration in Figs. 5 and 6. They are shown in each figure for dots at the left and for rings at the right. The upper panels show results for $\gamma=2 \mathrm{meV}$ and the lower panels for $\gamma$ $=5 \mathrm{meV}$. The crucial difference between dots and rings is in the crossing of energy levels. For dots there are no crossings for varying magnetic field strength $B$, opposite to the behavior of the rings. So the $\Psi$ data display quite monotonous ridgelike patterns for dots but a clear "hill/valley" pattern for rings. This represents a typical manifestation of the optical Aharonov-Bohm effect. This more remarked dependence of the rings upon changes in the magnetic field strength gives them better characteristics for practical use than dots.

This behavior becomes even more manifest for the other ellipsometric angle $\Delta$ as shown in Fig. 6. In degrees the peak to peak values for $\Delta$ are about five times larger than the corresponding variation in $\Psi$. Rings in general respond stronger for both angles than dots. This is remarkable, since the volume fraction $f_{V}$ is smaller for rings than for dots. Yet the understanding of this behavior is quite down to earth: the crossings cause two pairs to contribute in resonance simultaneously and this effectively doubles the response. All values drop by about the same factor as the damping $\gamma$ is increased for all cases. This is due and in agreement with the general behavior of the frequency dependent functions $f_{l k}(\omega)$, as given by Eq. (1). All values for $\Psi$ and $\Delta$ have variations of the order of magnitude of degrees and are therefore easily 

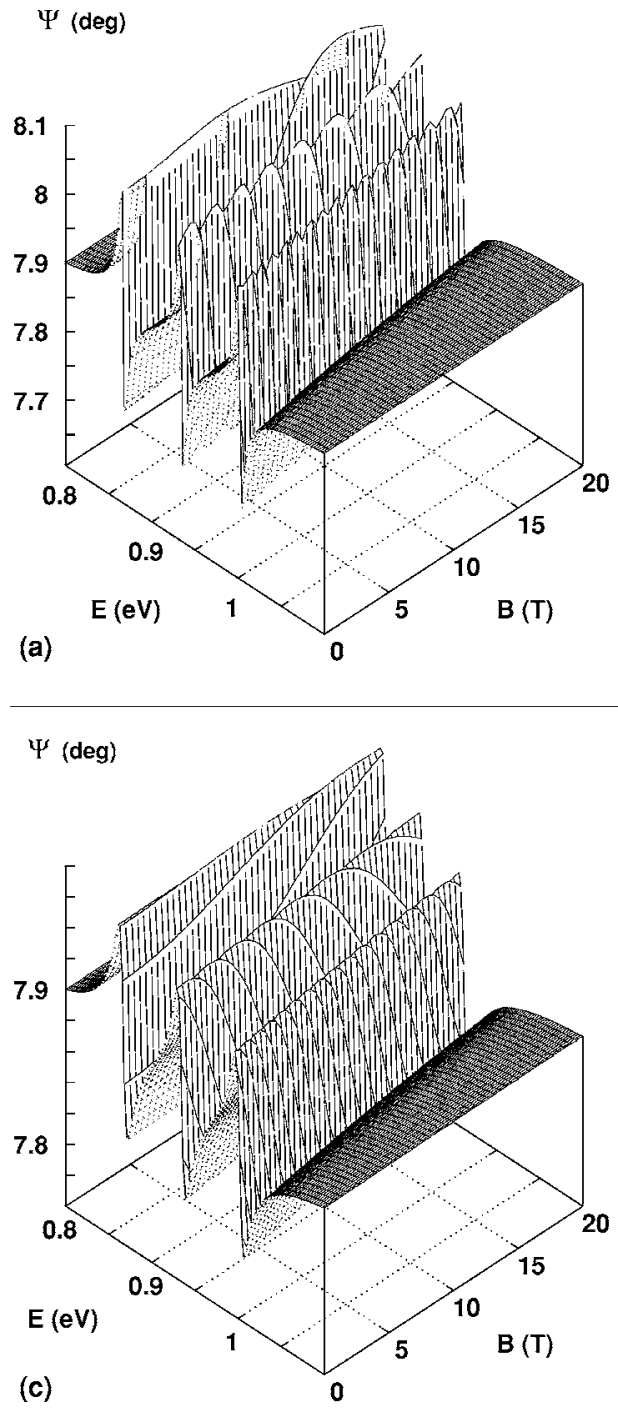
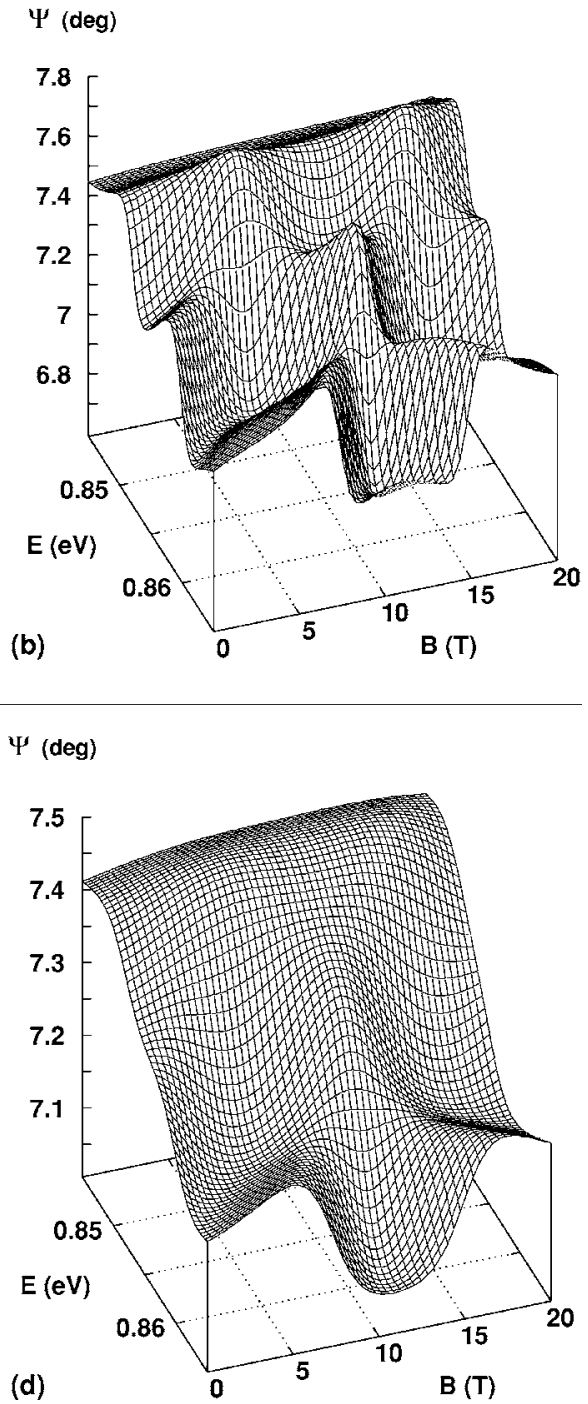

FIG. 5. Ellipsometric angle $\Psi$ for a monolayer of InAs quantum dots and nanorings. (a),(b) $\Psi$ for $\theta_{i}=60^{\circ}$ and $\hbar \gamma=2 \mathrm{meV}$. (c),(d) $\Psi$ for $\theta_{i}=60^{\circ}$ and $\hbar \gamma=5 \mathrm{meV}$. Left panels (a),(c): dots, right panels (b),(d): rings. within range of any modern ellipsometer. Yet it should be mentioned that the variations in $\Psi, \Delta$ will reduce drastically, when the embedding is taken into account also, as we hope to show in a forthcoming publication.

The ellipsometric parameters $\Psi, \Delta$ are relative quantities and do not contain knowledge about the absolute values of optical response coefficients. Therefore also reflectance and absorbance have been determined for a case where both the ring and the dot lattice have a maximum in the imaginary part of their polarizability (resonance). For both systems we chose a magnetic field strength of $B=7 \mathrm{~T}$ and a damping of $\hbar \gamma=5 \mathrm{meV}$. The selected resonance frequencies were $\hbar \omega$ $=0.867 \mathrm{eV}$ for the dot and $\hbar \omega=0.854 \mathrm{eV}$ for the ring lattice. The reflectance for those settings is shown in Fig. 7. Typically the values are in the $10^{-5}$ range, so pretty weak. The ring lattice has a slightly weaker reflectance than the dot lattice, but both systems display the usual angular dependent behavior. The most outspoken aspect is the occurrence for $p$ polarization of the Brewster minimum at about $68^{\circ}$. For bulk InAs the Brewster angle has the value of $74^{\circ}$ for comparison.

By means of Eq. (40) we have also calculated the absorbance. This quantity has the most direct connection to the detailed treatment of the microscopic behavior given in this paper, both electromagnetically and quantum mechanically. The results are shown in Fig. 8. The overall picture is that the absorbance in the ring system is stronger than for the dot system. This is the consequence of the enlargement at resonance of the imaginary part of the polarizability due to the crossing of energy levels in the ring system. Indeed the enlargement is about a factor of 2 . The most remarkable aspect of the absorbance is the strongly increased dichroism for increasing angles of incidence. For the hypothetical case that the dots or rings would have had an isotropic polarizability, both polarization directions $s$ and $p$ would behave similar to the $s$ components in Fig. 8 . Although the anisotropic behavior of the polarizability of the elements goes back both to electromagnetism (through the electromagnetic self-tensor) and to quantum mechanics (through the expectation value of the position vector), the dichroism is governed in the first place by quantum mechanics. For light components in the $z$ direction the dots and rings will be transparent, for the remaining $x, y$ direction they will be absorbing. So it looks as if research concerning the shape and volume independence of the optical response for isolated levels at resonance, should focus upon absorbance and the dichroism found here. 


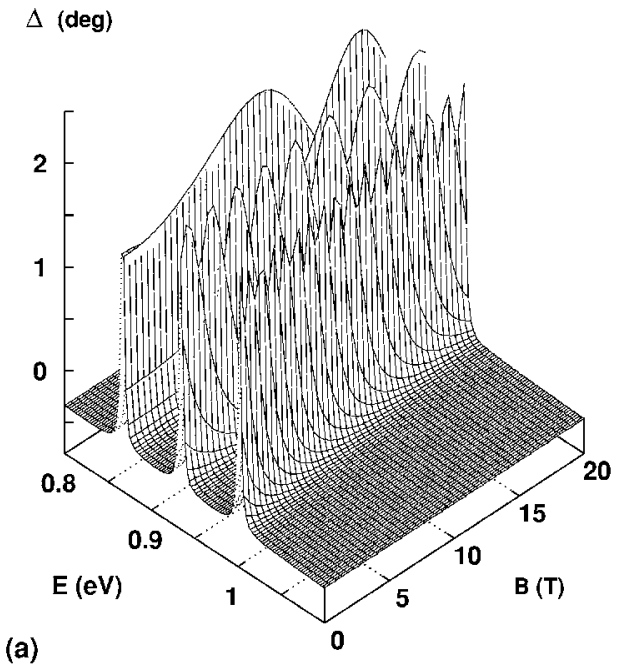

(a)

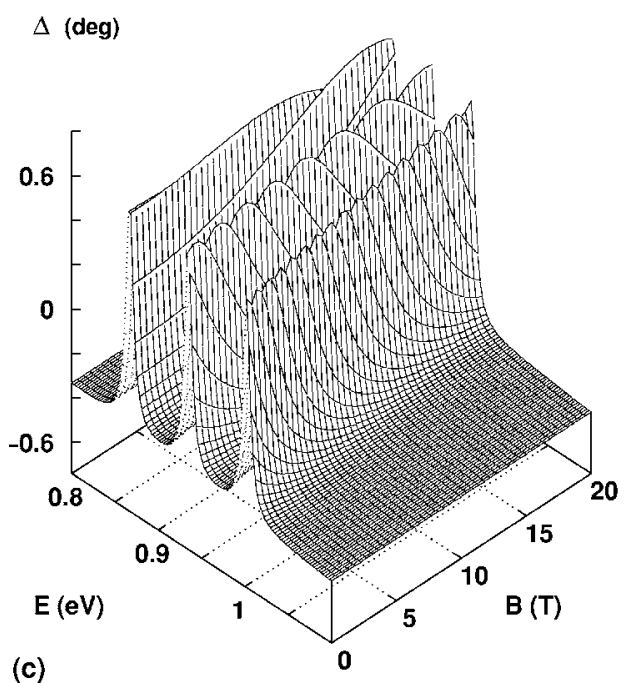

$\Delta$ (deg)

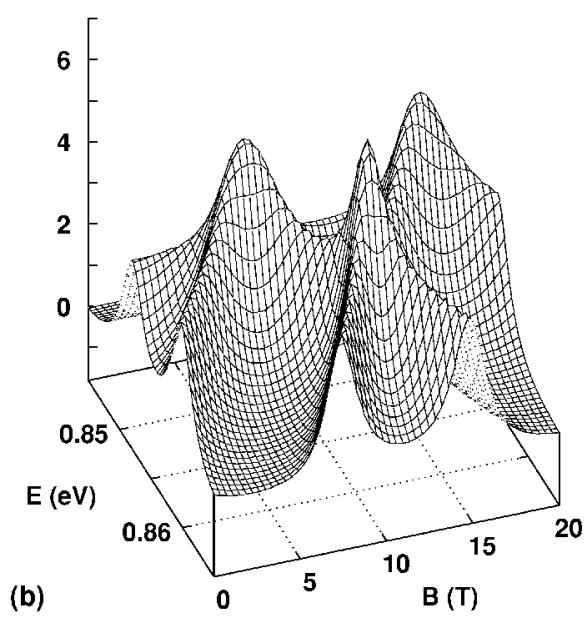

$\Delta$ (deg)

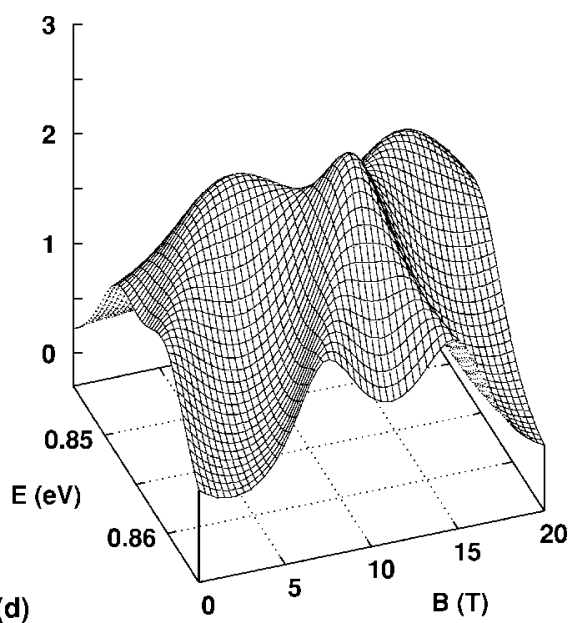

FIG. 6. Ellipsometric angles $\Delta$ for a monolayer of InAs quantum dots and nanorings. (a),(b) $\Delta$ for $\theta_{i}=60^{\circ}$ and $\hbar \gamma=2 \mathrm{meV}$. (c), (d) $\Delta$ for $\theta_{i}=60^{\circ}$ and $\hbar \gamma=5 \mathrm{meV}$. Left panels (a),(c): dots, right panels (b),(d): rings.

\section{SUMMARY AND CONCLUSIONS}

In this paper we have performed a comparative study of the optical response functions (such as reflectance and absorbance and the ellipsometric angles $\Psi, \Delta$ ) for quantum dots

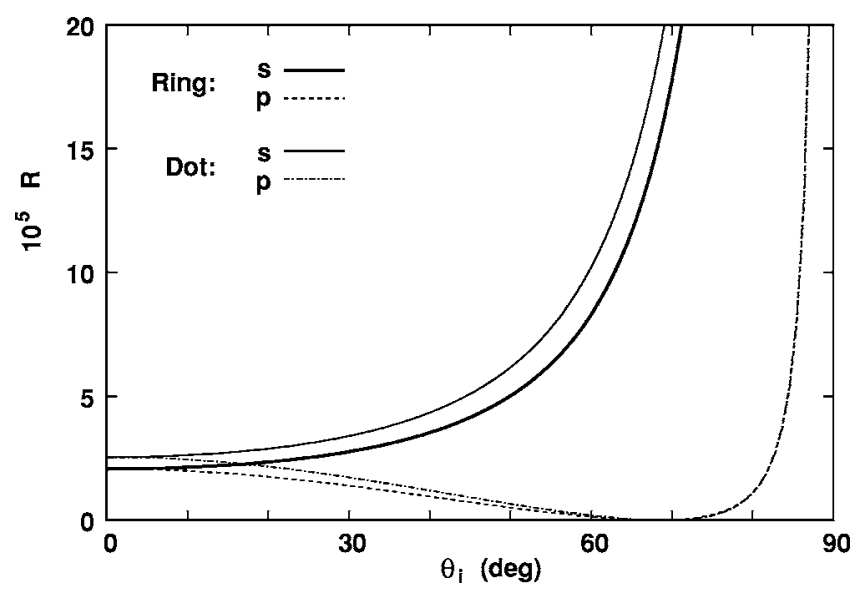

FIG. 7. Reflectance $R$ for a monolayer of InAs quantum dots and nanorings. $B=7 \mathrm{~T}$. Dots: $\hbar \omega=0.867 \mathrm{eV}$, rings: $\hbar \omega=0.854 \mathrm{eV}$. Both: $\hbar \gamma=5 \mathrm{meV}$. and nanorings, when they are arranged in a square lattice. We have developed accurate and workable expressions for the response terms of separate quantum dots and nanorings as given by the polarizability. The polarizability is determined

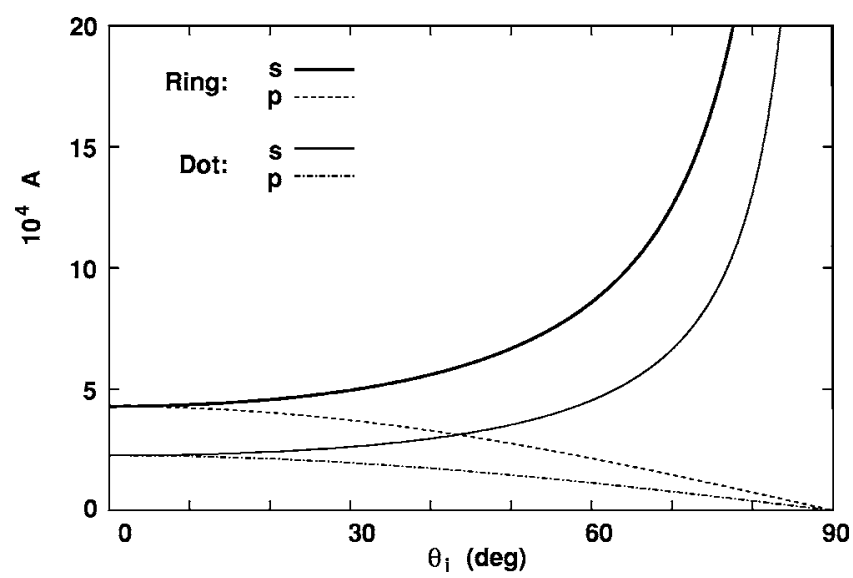

FIG. 8. Absorbance $A$ for a monolayer of InAs quantum dots and nanorings. $B=7 \mathrm{~T}$. Dots: $\hbar \omega=0.867 \mathrm{eV}$, rings: $\hbar \omega=0.854 \mathrm{eV}$. Both: $\hbar \gamma=5 \mathrm{meV}$. 
by both quantum-mechanical and electromagnetic interactions. For nano-objects an outspoken consequence of these two aspects of the polarizability is that far below the energy gap the strength of the polarizability is volume and shape dependent, whereas for separately observable resonant transitions at the absorption edge the strength is volume and shape independent. For the optical response of the square lattices made up from these nano-objects only the electromagnetic interaction needs to be taken into account. The remote response, as represented by reflection and transmission coefficients has been obtained by remote propagators as usual in discrete optics and these coeficients build a key instrument for the quantitative analysis of the magneto-optical response of lattices made from nanosized objects. The calculations clearly show that rings are more effective to exploit the dependence from magnetic fields than dots. Despite a lower volume fraction rings have stronger variation in any of the ellipsometric angles than the dots. The crossing of the transition energies, being characteristic for rings and known as the optical Aharonov-Bohm effect, results in a pronounced variation of the ellipsometric angles for varying magnetic field. The reflectances for both types of lattice are weak, as can be expected from such thin layerlike systems. Remarkable is the strongly increasing dichroism for increasing angles of incidence. Since the origin of this dichroism is in the dynamic part of the anisotropy of the polarizability of the nano-objects, this dichroism can be of use to investigate the size- and shape-dependent behavior of the polarizability. The theoretical findings obtained here, yield also the essential starting point for future work to incorporate the influence of the embedding (capped quantum-dot or nanoring systems). This comparative study shows that use of nano-rings or quantum dots in both the investigation and use of magnetooptical response is in favor of the first.

\section{ACKNOWLEDGMENTS}

This work was funded by the National Science Council of Taiwan under Contracts No. NSC-93-2215-E-009-006 and NSC-93-2112-M-009-008.
${ }^{1}$ J. D. Joannopoulos, R. D. Meade, and J. N. Winn, Photonic Crystals: Molding the Flow of Light (Princeton University Press, Princeton, 1995).

${ }^{2}$ V. G. Veselago, Sov. Phys. Usp. 10, 509 (1968).

${ }^{3}$ J. B. Pendry, A. J. Holden D. J. Robbins, and W. J. Stewart, IEEE Trans. Microwave Theory Tech. 47, 2075 (1999).

${ }^{4}$ O. Voskoboynikov, Yiming. Li, Hsiao-Mei Lu, Cheng-Feng Shih, and C. P. Lee, Phys. Rev. B 66, 155306 (2002); O. Voskoboynikov and C. P. Lee, Physica E (Amsterdam) 20, 278 (2004).

${ }^{5}$ D. Bimberg, M. Grundmann, and N. N. Ledentsov, Quantum Dot Heterostructures (Wiley Interscience, New York, 1998).

${ }^{6}$ J. M. Garcia, G. Medeiros-Ribeiro, K. Schmidt, T. Ngo, J. L. Feng, A. Lorke, J. Kotthaus, and P. M. Petroff, Appl. Phys. Lett. 71, 2014 (1997) .

${ }^{7}$ Y. Aharonov and D. Bohm, Phys. Rev. 115, 485 (1959).

${ }^{8}$ A. O. Govorov, S. E. Ulloa, K. Karrai, and R. J. Warburton, Phys. Rev. B 66, 081309(R) (2002).

${ }^{9}$ J. I. Climente, J. Planelles, and W Jaskolski, Phys. Rev. B 68, 075307 (2003); J. Climente, J. Planelles, W. Jaskolski, and J. I. Aliaga, J. Phys.: Condens. Matter 15, 3593 (2003).

${ }^{10}$ A. Lorke and R. J. Luyken, Physica B 256-B258, 424(1998); A. Lorke, R. J. Luyken, A. O. Govorov, J. P. Kotthaus, J. M. Garcia, and P. M. Petroff, Phys. Rev. Lett. 84, 2223 (2000).

${ }^{11}$ J. Planelles, W. Jaskolski, and J. I. Aliaga, Phys. Rev. B 65, 033306 (2001).

${ }^{12}$ M. Bayer, M. Korkusinski, P. Hawrylak, T. Gutbrod, M. Michel, and A. Forchel, Phys. Rev. Lett. 90, 186801 (2003).

${ }^{13}$ H. Pettersson, R. J. Warburton, A. Lorke, K. Karrai, J. P. Kotthaus, J. M. Garcia, and P. M. Petroff, Physica E (Amsterdam) 6, 510 (2000).

${ }^{14}$ G. Y. Slepyan, S. A. Maksimenko, V. P. Kalosha, A. Hoffmann, and D. Bimberg, Phys. Rev. B 64, 125326 (2001).

${ }^{15}$ G. P. M. Poppe, C. M. J. Wijers and A. van Silfhout, Phys. Rev. B 44, 7917 (1991).
${ }^{16}$ J. Vlieger, Physica (Amsterdam) 64, 63 (1973); C. M. J. Wijers and K. M. E. Emmett, Phys. Scr. 38, 435(1988).

${ }^{17}$ B. C. Lee, O. Voskoboynikov, and C. P. Lee, Physica E (Amsterdam) 24, 87 (2004).

${ }^{18}$ X. Z. Liao, J. Zou, X. F. Duan, D. J. H. Cockayne, R. Leon, and C. Lobo, Phys. Rev. B 58, R4235 (1998)

${ }^{19}$ H. A. Kramers and W. Heisenberg, Z. Phys. 31, 681 (1925).

${ }^{20}$ O. S. Stier, M. G. Grundmann, and D. B. Bimberg, Phys. Rev. B 59, 5688 (1999).

${ }^{21}$ G. W. Bryant, Phys. Rev. B 37, 8763 (1988).

${ }^{22}$ P. Enders, A. Bärwolff, M. Woerner, and D. Suisky, Phys. Rev. B 51, 16695 (1995).

${ }^{23}$ C. M. J. Wijers, Phys. Rev. A 70, 063807 (2004).

${ }^{24}$ H. E. van den Brom and J. M. van Ruitenbeek, Phys. Rev. Lett. 82, 1526 (1999).

${ }^{25}$ G. Bastard, Wave Mechanics Applied to Semiconductor Heterostructures (Les Edition de Physique, Les Ulis, 1990).

${ }^{26}$ J. I. Climente, J. Planelles, and J. L. Movilla, Phys. Rev. B 70, 081301(R) (2004).

${ }^{27}$ E. O. Kane, J. Phys. Chem. Solids 1, 249 (1957).

${ }^{28}$ M. Asada, A. Kameyama, and Y. Suematsu, IEEE J. Quantum Electron. 20, 745 (1984); M. Asada and Y. Suematsu, ibid. 21, 434 (1985); M. Asada, Y. Miyamoto, and Y. Suematsu, ibid. 22, 1915 (1986).

${ }^{29}$ L. V. Asryan and R. A. Suris, Semicond. Sci. Technol. 11, 554 (1996).

${ }^{30}$ S. L. Chuang, Physics of Optoelectronic Devices (Wiley, New York, 1995).

${ }^{31}$ P. G. Eliseev, H. Li, A. Stintz, G. T. Liu, T. C. Newell, K. J. Malloy, and L. F. Lester, Appl. Phys. Lett. 77, 262 (2000).

${ }^{32}$ V. Belevitch and J. Boersma, Philips J. Res. 38, 79 (1983).

${ }^{33}$ J. Avelin, Ph.D. thesis, Electromagnetics Laboratory, Helsinki University of Technology, 2003. 